\title{
GEOPLANO: UMA ABORDAGEM INCLUSIVA
}

\author{
GEOPLAN: AN INCLUSIVE APPROACH
}

Walber Rodrigues de Assis ${ }^{1}$

Amaya de Oliveira Santos ${ }^{2}$

\begin{abstract}
RESUMO: O vigente trabalho visa apresentar um estudo sobre as contribuições de materiais concretos no que concerne a inclusão de discentes com deficiência visual no ambiente escolar, no qual, irá discorrer sobre as implicações da utilização do geoplano como ferramenta didático-pedagógico para o auxílio do ensinoaprendizagem de conteúdos matemáticos. A metodologia empregada teve aspecto bibliográficoexperimental, o qual, buscou investigar a literatura científica relacionada com o tema proposto, bem como, examinar as peculiaridades do geoplano na discussão sobre temas elementares da geometria euclidiana plana. Com isso, pode-se compreender melhor, como este material contribui para o ensino-aprendizagem de matemática dentro de uma proposta inclusiva de modo a enriquecer as experiências em sala de aula.
\end{abstract}

Palavras-chave: Ensino. Geometria. Geoplano. Inclusão.

ABSTRACT: The present work aims to present a study on the contributions of concrete materials with regard to the inclusion of students with visual impairment in the school environment, which will discuss the implications of using the geoplane as a didactic-pedagogical tool to aid teaching. learning mathematical content. The methodology employed was a bibliographic-experimental aspect, which sought to investigate the scientific literature related to the proposed theme, as well as to examine the peculiarities of the geoplane in the discussion of elementary themes of flat Euclidean geometry. With this, one can better understand how this material contributes to the teaching and learning of mathematics within an inclusive proposal in order to enrich classroom experiences.

Keywords: Teaching. Geometry. Geoplane. Inclusion.

\section{INTRODUÇÃO}

O conhecimento matemático, por mais elementar que seja, seguramente traz benefícios para quem os tem. A exemplo disso, as noções geométricas são de grande utilidade quando lidamos com formas, tanto, que suas características são exploradas pelos discentes desde cedo, visto que “[...] constituem parte importante do currículo de Matemática no ensino fundamental, porque, por meio deles, o aluno desenvolve um tipo especial de pensamento que lhe permite compreender, descrever e representar, de forma organizada, o mundo em que vive"(BRASIL, 1997, p.39).

Quando discutimos sobre inclusão, a questão não é diferente, sendo um conhecimento importante por estar presente em diversos aspectos do dia-a-dia. Entretanto, pessoas com deficiência visual, por exemplo, certamente enfrentam maiores dificuldades na sua inserção em sala

\footnotetext{
${ }^{1}$ Acadêmico de Licenciatura em Matemática pelo Instituto Federal do Piaú - IFPI, Campus São Raimundo Nonato;

2 Professora do Ensino Básico, Técnico e Tecnológico - Licenciatura/Disciplina pedagógicas / LIBRAS do Instituto Federal do Piauí - IFPI, Campus São Raimundo Nonato e Pesquisadora no Grupo de Pesquisa Inclusão e

Diversidade na área de Educação Especial, Educação Inclusiva e Libras.
} 
de aula, isto considerando-se que é comum, professores explorarem recursos visuais em suas metodologias. Ao se pensar no ensino-aprendizagem de matemática, a realidade não é diferente, uma vez que a utilização de imagens como desenhos geométricos e gráficos, em geral, costuma facilitar a compreensão das abstrações desta disciplina e de certo modo, trata-se de uma estratégia acessível do ponto de vista prático e financeiro.

Nestas circunstâncias, a adoção de materiais concretos apropriados pode contribuir significativamente para minimizar estas dificuldades, pois, além da ilustração, tem-se ainda o tato. Com isso esses materiais atuam como auxílio tanto para deficientes visuais quanto para discentes com boa visão e por consequência, servem também como apoio a inclusão, uma vez que permite a construção de conhecimentos teóricos e até mesmo valores humanos básicos.

\section{METODOLOGIA}

O trabalho em questão seguiu, como assim destaca Marconi e Lakatos (2003), pelo método bibliográfico-experimental, uma vez que, este possibilita a revisão da literatura existente, além de familiarizar o pesquisador com o objeto de estudos. Para tanto, o desenvolvimento ocorreu em três etapas conforme listadas a seguir.

Primeiramente, realizou-se um levantamento bibliográfico sobre os temas: deficiência visual; ensino-aprendizagem de matemática, em especial, sobre alguns aspectos elementares da geometria euclidiana plana; além do instrumento didático-pedagógico geoplano.

A etapa seguinte, foi destinada para a construção do material, no qual, foram construídos dois geoplano, utilizando-se para isso, materiais de baixo custo financeiro e reciclados. Por fim, deu-se a uma análise experimental mediante um estudo de caso sobre as particularidades do item produzido, bem como, suas contribuições para o ensino-aprendizagem de matemática em uma perspectiva de inclusão de alunos com deficiência visual.

\section{REFERENCIAL TEÓRICO}

\section{Deficiência Visual}

A deficiência visual como diz Campos, Sá e Silva (2007), consiste na perda parcial ou total da visão. No primeiro grupo estão presentes uma variedade de níveis de perdas visuais que podem 
ser leve, moderadas ou profundas, mas que ainda podem ser minimizadas mediante tratamento adequado. No entanto, “a cegueira é uma alteração grave ou total de uma ou mais das funções elementares da visão que afeta de modo irremediável a capacidade de perceber cor, tamanho, distância, forma, posição ou movimento em um campo mais ou menos abrangente” (p.15).

Ao refletir sobre esse tema, Gil (2000) utiliza o termo visão subnormal para referir-se, aos casos de perda parcial da visão, o qual, define como uma "alteração da capacidade funcional decorrente de fatores como rebaixamento significativo da acuidade visual, redução importante do campo visual e da sensibilidade aos contrastes e limitação de outras capacidades” (p. 6).

A referência, imediatamente anterior, salienta ainda que entre os fatores que podem causar perdas visuais, estão as patologias, como miopia, estrabismo, astigmatismo, ambliopia, hipermetropia. Estas necessitam de cuidados, uma vez que podem interferir nas atividades cotidiana, como por exemplo, no desenvolvimento e na aprendizagem.

Conforme dados do IBGE (2010) e divulgados pelo site da Fundação Dorina [201-], 3,5\% da população brasileira possui alguma deficiência visual, isso corresponde a cerca de 6,5 milhões de pessoas, no qual, 528.624 tem perda total da visão, 6.056.654 possuem alguma dificuldade para enxergar. Desse número, a região nordeste do Brasil está em segundo lugar com 2.192.455 pessoas com deficiência visual, ficando atrás apenas da região Sudeste com 2.508.587. As regiões Norte, Sul e Centro-Oeste, aparecem respectivamente com 574.823, 866.086 e 443.357.

Estes dados ainda revelam que em termos de porcentagem da população local, a região nordeste é a que mais possui pessoas com deficiência visual com 4,1\% dos indivíduos. As demais posições são ocupadas pelas regiões Norte 3,6\%, Sul e Centro-Oeste ambas com 3,2\% e por último a região Sudeste com $3,1 \%$ de sua população.

\section{Ensino de Matemática para Deficientes Visuais}

Ao examinar o processo de ensino-aprendizagem envolvendo pessoal com algum grau de deficiência visual, Campos, Sá e Silva (2007) destacam a importância de considerar na estratégia a ser empregada fatores como: nível de aceitação; implicações decorrentes da deficiência; desenvolvimento dos demais sentidos, audição, olfato, paladar e tato; como também, os recursos didáticos disponíveis.

Essas autoras apontam ainda, que a utilização de recursos para este público deve priorizar a evolução dos demais sentidos. Neste cenário, tornar-se importante distinguir as diferenças para que se possa produzir ou adequar materiais, de tal modo, que se valorize o ensino-aprendizagem no contexto da inclusão. 
Assim, Nery (2013) vai apontar que uma deficiência como a visual, não deve de forma alguma ser empecilho para o ensino-aprendizagem, sobretudo na área de matemática onde comumente se recorre a abordagens visuais. Aqui, a utilização dos materiais concretos contribui para facilitar a participação e compreensão de conceitos abstratos e desse modo, auxilia na construção do conhecimento matemático.

\section{Geoplano para Auxílio no Ensino - Aprendizagem de Matemática no Contexto da Inclusão de Discentes com Deficiência Visual.}

O Geoplano é um material concreto didático para o auxílio do ensino-aprendizagem de matemática, sobretudo, no que diz respeito a geometria plana. Seu tabuleiro é construído sob superfície plana, onde são fixados em linhas paralelas de mesmo espaçamento, pregos ou similares.

Moraes et al (2008) destaca que este jogo foi desenvolvido por Caleb Gattegno em 1961 com finalidades didáticas visando o estudo de geometria plana, no qual, sua utilização possibilita a construção de atividades acessíveis, destinadas ao:

Trabalho com a lateralidade; A identificação e reprodução de figuras geométricas; A identificação e diferenciação de unidades de medida; A compreensão das ideias de semelhança e congruência; A identificação e comparação de propriedades de figuras; A produção de figuras semelhantes a outras dadas; A medição e comparação de áreas e perímetros para a compreensão das diferenças entre tais conceitos [...] (p.2).

Silva (2014) relata que "o geoplano assim como outros materiais manipuláveis, oferece um apoio mental, facilitando aos alunos a abstração de conceitos algébricos ou geométricos e auxiliando no desenvolvimento de vários conteúdos da disciplina de matemática” (p.17). A autora aponta ainda para a existência de uma diversidade extensa desse material e entre os mais comuns estão as versões adaptadas para o ensino-aprendizagem de temas relacionados a geometria euclidiana plana e as versões com sistemas de coordenadas cartesianas que permite representar funções elementares, distâncias etc.

Por ser um material manipulável, permite que pessoas com deficiência visual, por exemplo, tenha acesso aos conteúdos de diversas áreas da matemática, como também, pode ser usado para auxílio de discentes sem deficiência.

\section{Figuras Geométricas Planas}

Esse tópico será dedicado ao estudo de alguns temas elementares que podem ser abordados com auxílio do geoplano. A exposição dará de forma abstrata para evidenciar algumas 
características relevantes sobre figuras euclidianas notáveis. Para este estudo será considerado como base, as pesquisas referentes ao trabalho de Dolce e Pompeo (2013).

Em geometria euclidiana plana, alguns entes caso do ponto, reta e plano, representados no diagrama da figura 01, são admitidos sem definição, ou seja, são aceitos como noções primitivas. Esses entes serão o ponto de partida para estudarmos algumas figuras planas, tais como, triângulos e quadriláteros.

Figura 01: representação gráfica dos entes ponto, reta e plano.

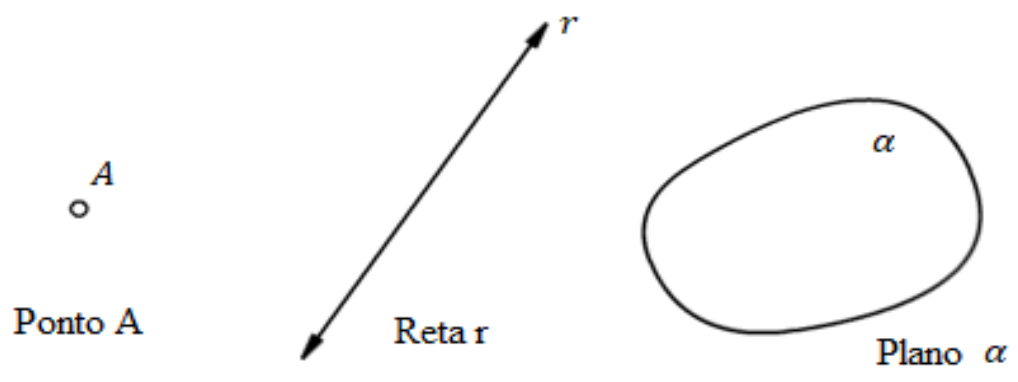

Fonte: dados da pesquisa.

No plano $\alpha$, da figura 01, as "bordas" servem apenas para facilitar a visualização, no entanto, o plano deve ser entendido como um ente bidimensional com dimensões infinitas. Semelhante a isso, a reta $r$, trata-se de um ente geométrico unidimensional de dimensão infinita, entretanto, se desta reta escolhermos dois pontos distintos $A$ e $B$, esses pontos reunidos com o conjunto de pontos que estar entre esses dois pontos, darão origem a um segmento de reta de extremos $A$ e $B$. A figura 02 expõe um segmento de reta $\overline{A B}$.

Figura 02: segmento $\overline{A B}$.

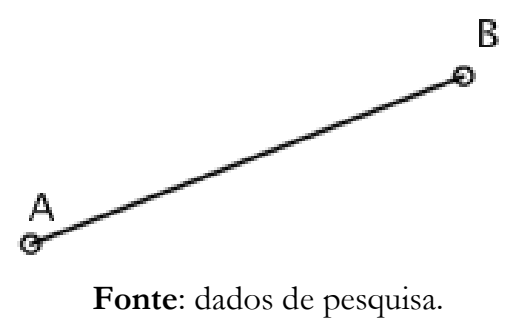

Agora consideremos, três pontos distintos $A, B$ e $C$, não colineares, isto é, não estão todos sobre a mesma reta, chama-se triângulo $A B C$, à região interna delimitada pela reunião dos segmentos $\overline{A B}, \overline{A C}$ e $\overline{B C}$, no qual, os pontos são identificados como vértices e os segmentos como lados do triângulo. Na figura 03 estar representado um exemplo de triângulo $A B C$.

Figura 03: triângulo $A B C$. 


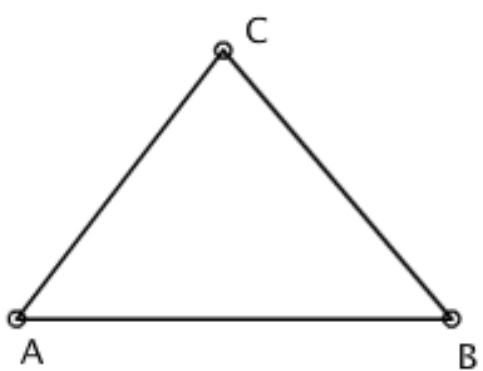

Fonte: dados da pesquisa.

Suponhamos agora, que dispomos de quatro pontos distintos $A, B, C$ e $D$ sendo três a três não colineares, nos quais, os segmentos $\overline{A B}, \overline{B C}, \overline{C D}$ e $\overline{D A}$ interceptam-se apenas nas extremidades. Nessas condições, denomina-se quadriláteros $A B C D$, à reunião desses segmentos. $\mathrm{Na}$ figura 04, são identificados dois tipos de quadriláteros: côncavo (ou não convexo), quando ao prolongar um dos segmentos este interceptar algum dos outros segmentos e caso contrário, o quadrilátero é dito convexo.

Figura 04: quadriláteros $A B C D$ convexo e côncavo.
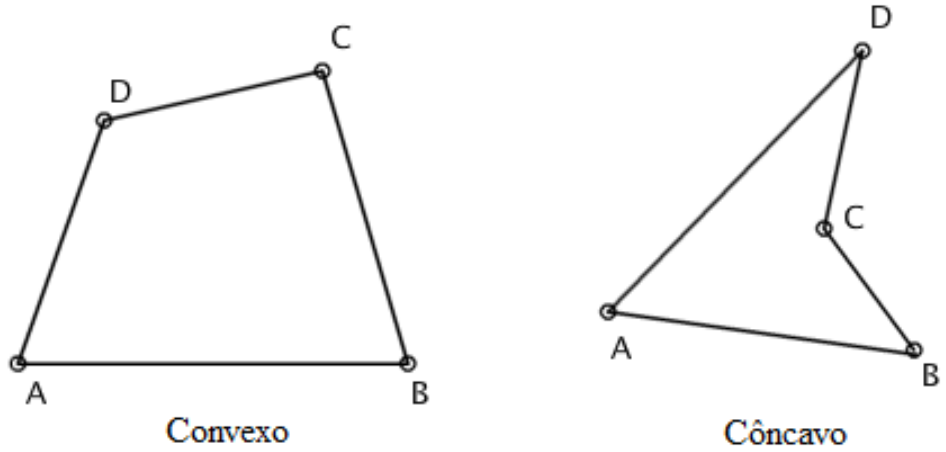

Fonte: dados da pesquisa.

Alguns quadriláteros convexos são de notáveis estudos na geometria plana, são eles: trapézio, quando dois dos segmentos, $\overline{A B}$ e $\overline{C D}$ ou $\overline{A D}$ e $\overline{B C}$ são paralelos; paralelogramo, se os segmentos opostos são paralelos; retângulo, possui todos os ângulos com mesma medida; losango, tem os quatro segmentos com mesma medida; quadrado, quando todos os lados e ângulos são congruentes, ou seja, possuem a mesma medida. A figura 05 ilustra os cinco (05) quadriláteros notáveis.

Figura 05: quadriláteros notáveis.

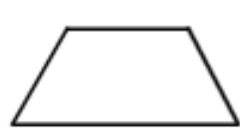

Trapézio

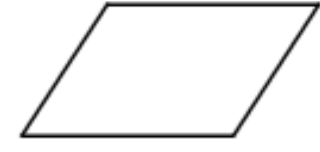

Paralelogramo

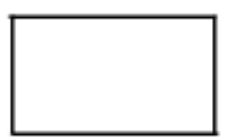

Retângulo

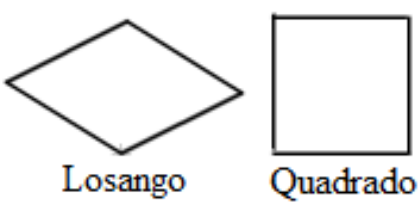

Quadrado

Fonte: dados da pesquisa. 
Essas figuras geométricas serão abordadas através do geoplano. Isso dará através de experimento por meio de um estudo de caso com estudante de baixa visão.

\section{RESULTADOS E DISCUSSÕES}

\section{Construção de um Geoplano}

Para construir um geoplano, são necessários: um conjunto de pinos, estes podem ser pregos ou similares, e uma base, quadrada de material resistente onde são pregados os pinos. Sob a base, desenha-se um conjunto de retas paralelas de mesmo espaçamento na horizontal e na vertical de modo a formar uma malha quadriculada, onde os pinos sejam pregados nas intersecções.

Neste trabalho, foram produzidos dois (2) geoplano. Na construção do primeiro material, foram utilizados papeis rígidos para a base, que teve dimensões de $16 \times 16 \mathrm{~cm}$. Nesta base, desenhou-se uma malha quadriculada, sendo, cada quadrado com medidas de $2 x 2 \mathrm{~cm}$. Sob as intersecções, fixou-se 81 alfinetes coloridos tipo tacha de modo a formar um quadrado de $8 x 8$ alfinetes. Para o desenvolvimento das atividades, foram utilizados elásticos de $8 \mathrm{~cm} \times 1,2 \mathrm{~mm} \times 1,5 \mathrm{~mm}$.

Para o segundo geoplano, utilizou-se, madeira para a base com dimensões de $22 x 22 \mathrm{~cm}$. A malha quadriculada tinha medidas de seus quadrados de $2 \times 2 \mathrm{~cm}$. Semelhante ao anterior, foram fixados 100 pregos de modo a formar um quadrado de $10 \times 10$. Na figura 06 , estão ilustrados os respectivos geoplano. 
Figura 05: Os dois geoplanos construídos para este trabalho.

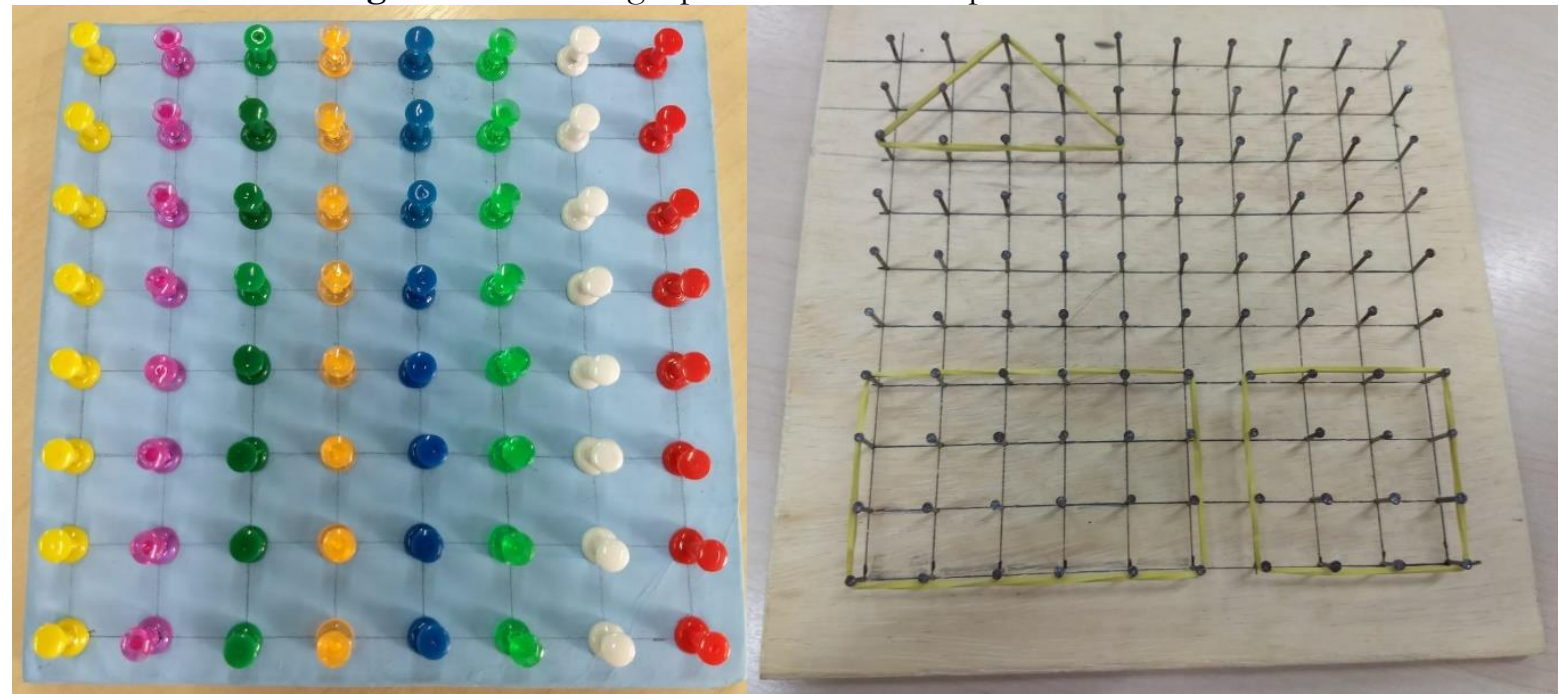

Fonte: dados da pesquisa.

Vale salientar que a escolha dos materiais ocorreu de modo a minimizar o gasto financeiro. Entretanto, pode-se utilizar diversos materiais, tais como: madeira, pregos, etc. Ficando a gosto do construtor.

\section{Experiência com o Geoplano}

A experiência ocorreu com uma voluntária de baixa visão, que cursa o primeiro módulo da licenciatura em física pelo Instituto Federal de Educação, Ciência e Tecnologia do Piauí - IFPI, Campus São Raimundo Nonato. O principal objetivo dessa etapa era identificar pelo tato, características de triângulos e quadriláteros notáveis: paralelogramo, retângulo, trapézio, quadrado, losango. Como critério de avaliação, foi observado a interação da discente com o material e o tema proposto, além da capacidade de executar as atividades sugeridas.

Figura 06: roteiro da atividade.

a) Dados os quadriláteros no geoplano, identifique os vértices, as arestas e classifique-os.

b) Desenhe no Geoplano:

i. Três retas paralelas sendo duas delas coincidentes.

ii. Quatro retas paralela e uma corrente.

iii. Retângulo de 4 e 2 unidades de medida.

iv. Uma figura de quatro lados congruentes.

v. Um trapézio de bases 6 e 3 unidades de medida.

vi. Dois triângulos com base medindo 3 e 4 unidades de medida.

vii. Quadrado de lados com três unidades de medida.

Fonte: dados da pesquisa.

A experiência ocorreu do seguinte modo: inicialmente, fez-se uma revisão sobre as características de retas, segmentos, triângulos e dos quadriláteros notáveis. Em seguida, a participante ficou de olhos vendados e foi-lhe pedido para identificar usando apenas o tato, alguns 
quadriláteros construídos previamente no geoplano, e suas características, tais como, vértices, arestas e dá suas respectivas classificações. Nesta etapa, a aluna através do tato, conseguiu distinguir os quadriláteros, bem como, identificar e conceituar as caraterísticas dos quadriláteros desenhado.

O segundo exercício constituiu de o participante desenhar sob o geoplano alguns quadriláteros, dados, algumas características, como, medida das arestas e classificação do quadrilátero. Além disso, pediu-se para construir triângulos e alguns segmentos de retas, dada sua medida ou se era paralelo ou concorrente a outra, conforme o item b do roteiro da figura 06.

Após a atividade, indagou-se sobre a impressões da aluna sobre os materiais utilizados, no qual, a mesma relatou que o geoplano confeccionado com madeira e pregos, devido ter um espaçamento maior entre os pinos (pregos), permite distinguir com maior facilidade através do tato, as caraterísticas das figuras, sobretudo com relação as medidas das arestas e se alguma figura possui lados em comum. Relata ainda que usaria em sala para introduzir outros conteúdos, tais como vetores, conceito de força, etc.

\section{CONSIDERAÇÕES FINAIS}

A experiência com o geoplano possibilitou conhecer com mais detalhes, algumas potencialidades desse instrumento. Neste estudo, ficou perceptível que o material dá um apoio significativo ao abordar assuntos matemáticos, em especial, a geometria plana.

Dentre as vantagens de se utilizar este material, destacamos as ilustrações que podem ser exploradas visualmente ou por meio do tato, onde pode ser abordados conteúdos iniciais ou até mesmo questões um pouco mais avançadas. Ainda existe a possibilidade de utilizá-lo como recurso lúdico para introduzir um assunto ou para resolver exercícios. Neste estudo de caso, por exemplo, foi possível rever questões a respeito de retas paralelas e concorrentes, além dos quadriláteros notáveis e algumas de suas características como, classificação, medida das arestas e identificação de seus vértices.

Como visto, as atividades no geoplano podem ser resolvidas sem ajuda visual, simplesmente com uso do tato, é possível extrair as informações desejadas das figuras. Além do mais, pode-se construir figuras geométricas planas, sendo conhecido algumas de suas características.

Por isso, um diferencial desse instrumento, é a possibilidades de dosar atividades visuais e de contato, além de ser um material viável para aquisição. Assim, esse instrumento pode ser utilizado dentro de um ambiente escolar no contexto da inclusão, o qual, seguramente trará contribuições positivas para o desenvolvimento do ensino-aprendizagem de matemática, além de valorizar a abordagem em sala de aula.

\section{REFERÊNCIAS}

BRASIL. Secretaria de Educação Fundamental. Parâmetros curriculares nacionais: matemática. Brasília: MEC/SEF, 1997.

CAMPOS, Izilda Maria de; SÁ, Elizabet Dias de; SILVA, Myriam Beatriz Campolina. Atendimento Educacional Especializado: deficiência visual. São Paulo: MEC/SEESP, 2007.

DE MORAES, Marcela Balbino Santos et al. Geoplano: Um Jogo Educacional Inteligente Para o Ensino de Geometria Plana. 2008. International Conference on Engineering and Technology Education São Paulo, BRAZIL, 2018. P. 559 - 563. 
DOLCE, Osvaldo; POMPEO, José Nicolau. Fundamentos de matemática elementar, 9: Geometria Plana. 9.ed. São Paulo: Atual, 2013.

FUNDAÇÃO DORINA. Sobre deficiência visual no Brasil. 201-. Disponível em: $<$ https://www.fundacaodorina.org.br/a-fundacao/deficiencia-visual/estatisticas-da deficiencia-visual/>. Acesso em: 28 de nov. de 2018.

GIL, Marta (org.). Deficiência visual. Brasília: MINISTÉRIO DA EDUCAÇÃO - MEC. Secretaria de Educação a Distância, 2000.

MARCONI, Marina de Andrade; LAKATOS, Eva Maria. Fundamentos de metodologia científica. 5. ed. São Paulo: Atlas, 2003.

NERY, Marcos Wildson Alves. Um olhar sobre a Educação Inclusiva de Deficientes Visuais: Estratégias de Ensino de Trigonometria e Geometria Espacial. Dissertação de mestrado submetida à Coordenação do Curso de Pós-Graduação em Matemática da Universidade Federal do Piauí, 2013.

SILVA, Joelma Alves da. Um estudo sobre os conceitos de área e perímetro por meio da utilização do Geoplano. Trabalho de Conclusão de Curso (Graduação em matemática) Universidade Estadual da Paraíba, Centro de Ciências e Tecnologia, 2014. 